\title{
A Simple Derivation of Crooks Relation
}

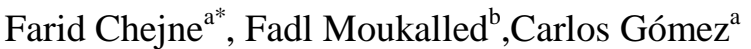 \\ ${ }^{a}$ TAYEA, Research Group, Facultad de Minas, Universidad Nacional de Colombia, Medellín, Colombia \\ ${ }^{\mathrm{b}}$ Faculty of Engineering and Architecture, American University of Beirut, Beirut, Lebanon \\ fchejne@unal.edu.co
}

\begin{abstract}
:
The link between properties of a system at equilibrium, in particular free energy difference, to the fluctuations in the work performed during non-equilibrium process is called Crooks relation. This relation, which is a measure of the grade of irreversibility of a process, was elegantly derived based on the equations of motion for a set of particles along with the formal solution of the evolution equation using a distribution function, both solved in a classical and a stochastic way. This technical note, reports on a simple derivation of Crooks formula based on the energy balance and entropy generation in a system undergoing a process in which fluctuations are not neglected
\end{abstract}

\section{Keywords: Crooks relation; fluctuations; far from equilibrium; rare events.}

\section{Introduction}

A macroscopic system is driven away from equilibrium if it is in contact with a heat reservoir, with the second law of thermodynamics acting as a limiter on the amount of work that can be performed on or by the system. On average, this work can never be larger than the change in the system's free energy. For a microscopic system the situation is different, as the thermal fluctuations give rise to a statistical distribution of work values with variation in time of any thermodynamic property being of the same order of magnitude as the physical dimensions of the system. There are several equations relating properties of a system at equilibrium to the fluctuations in the work performed during a non-equilibrium process. One of the most important fluctuation relations is known as the Crooks relation [1]; it allows for the measurement of the degree of irreversibility in a process.

Fluctuations always exist in any kind of system, whether at equilibrium or far from equilibrium, and are due to the random motion of particles known as the Brownian motion. The impacts caused by the random motion of molecules generally result in two kinds of effects: (i) they act as a random driving force to the Brownian particles, and (ii) they give rise to a frictional force that affects the motion [1].

The fluctuations (the irregular molecular collisions) in a system at the macroscopic scale are responsible for the linear transport phenomena [2-5], while at the microscopic scale they are the cause of the rare events that violate the second law of thermodynamics (e.g. negative heat flux). In general, as the size of a system decreases the role of fluctuations increases; therefore, it is reasonable to assume that such rare events can be observed in systems that are small, e.g. thermal conduction in a nanotube whose extremities are connected to two heat reservoirs [6]. This indicates that the physics on a Nano scale could be different from that at a macroscopic scale. In particular, large fluctuations occur at the Nano scale, with mostly unknown consequences [7].
Therefore, the second law of thermodynamics can be verified only on average because entropy production can have negative instantaneous values. Systems that are not in equilibrium are characterized by an irreversible heat transfer between their boundaries and the environment (the thermal bath). For such systems, the direction of heat transfer and the probability of having either positive or negative entropy production have to be calculated using one of the fluctuation theorems (FTs) [8].

For a system to be considered in thermodynamic equilibrium, the net heat flux between its boundaries and the surroundings has to be zero. In this case, equal amounts of heat are absorbed and released at all times causing the small fluctuations, due to the Brownian motion, to die out instantly through dissipation (the viscous drag). For a system to be considered not far from thermodynamic equilibrium the ratio between the heat absorbed and that which is released is equal to 1 at steady state; but the heat flux between the system and its surroundings cannot be zero. This situation is akin to the fluctuation-dissipation phenomenon where a system receives information from the outside to intensify fluctuations, and through the transportation of this information these fluctuations weaken, (i.e. are dissipated).

On the other hand, if a system is far from equilibrium, this ratio between the absorbed and released heat is different than 1 and the information received by the system, from the outside, is not entirely absorbed. The difference between the heat rejected and absorbed gives rise to what is called rare events, which are responsible for the local violation of the second law of thermodynamics. For such systems, internal fluctuations are non-uniform creating complicated interconnected effects that lead to rare events. An example could be a heat flux flowing in a direction opposite to that established by the second law of thermodynamics.

Understanding these rare events led to the development of the so called fluctuation theorems (FTs) by Evans and Searle's for systems evolving from equilibrium toward a non-equilibrium steady state [9], and by Gallavoti and 
Cohen for steady-state systems [10-23]. Predictions with these models show that systems can reject/absorb more heat than they can absorb/reject thereby revealing the rare events that locally violate the second law of thermodynamics.

The amount of heat exchanged or dissipated $(\psi=Q)$ at steady state between a non-equilibrium system and a heat reservoir is equal to the entropy production $\left(S_{g e n}\right)$ multiplied by the temperature $(\mathrm{T})$ and is computed as

$\psi=T S_{g e n}=Q$

For systems that are not in thermodynamic equilibrium, the probability for entropy production to be either positive $[P(\psi)]$ or negative $[P(-\psi)]$ is quantitatively governed by the Fluctuation Theorem (FT). An explicit mathematical expression for the ratio of probabilities, $P(\psi) / P(-\psi)$, validated for non-equilibrium systems at steady state, is given by

$$
\frac{P(\psi)}{P(-\psi)}=\exp \left(\frac{\psi}{k_{B} T}\right)
$$

The above expression indicates that at steady-state, the probability for heat to be lost by the system (positive $\psi$ ) is higher than the probability for heat to be gained by the system (negative $\psi$ ) under the same conditions. Therefore, it can be concluded that on average at steady state, systems far from thermodynamic equilibrium always dissipate (lose) heat. Moreover, heat being an extensive property, the ratio of probabilities $[P(\psi) / P(-\psi)]$ increases exponentially with the system size indicating that the probability for heat to be absorbed by macroscopic systems is insignificant.

C. Jarzynski [24] reported on a work relationship for systems experiencing non-equilibrium processes called the Jarzynski equality (hereafter referred to as JE). The JE states that for any system going through a non-equilibrium process from the initial state of equilibrium of $\mathrm{A}$, to a final equilibrium state of $\mathrm{B}$, the following relation holds:

$\exp \left(-\frac{\Delta G}{k_{B} T}\right)=\left\langle\exp \left(-\frac{W}{k_{B} T}\right)\right\rangle$

In Eqn (3), $\Delta G$ is the free-energy difference between the equilibrium states, $A$ and $B, k_{B}$ is the Boltzmann constant, $T$ is the temperature of the heat reservoir, and the average $\langle\ldots\rangle$ is taken over an infinite number of repeated nonequilibrium experiments carried out with the protocol $\mathrm{x}(t)$. Denoting the dissipated work along a given trajectory by $\left\langle W_{\text {dis }}\right\rangle$, with its value computed as

$$
\left\langle W_{d i s}\right\rangle=W-\Delta G
$$

and combining Jensen's inequality [17] given by

$$
\langle\exp (x)\rangle \geq \exp (\langle x\rangle)
$$

with the JE equality [equation (3)], the following version of the second law of thermodynamics can be derived as

$$
\begin{aligned}
& \left.\exp \left(-\frac{\Delta G}{k_{B} T}\right)=\left\langle\exp \left(-\frac{W}{k_{B} T}\right)\right\rangle\right\} \\
& \langle\exp (x)\rangle \geq \exp (\langle x\rangle) \\
& \Rightarrow \exp \left(-\frac{\Delta G}{k_{B} T}\right) \geq \exp \left(-\frac{\langle W\rangle}{k_{B} T}\right) \Rightarrow\left\langle W_{d i s}\right\rangle \geq 0
\end{aligned}
$$

For the equality to hold, it is necessary for the energy dissipated to have negative values, which is a violation of the second law of thermodynamics. Using the reversible equations of motion to demonstrate the above relation, it was found that the second law of thermodynamics holds on average; and there must always exist non-equilibrium trajectories with $W_{d i s}=0$ for the equality to hold. These trajectories, sometimes referred to as transient violations of the second law, represent large fluctuations in the work which serve to ensure that the equations of motion at the microscopic level are time-reversal invariant.

G. E. Crooks [2-4] derived a generalized fluctuationdissipation equation for stochastic microscopically reversible dynamics given by

$$
\frac{P_{F}(W)}{P_{R}(-W)}=\exp \left(-\frac{W-\Delta G}{k_{B} T}\right)
$$

where $P_{F}(W)$ and $P_{R}(-W)$ stand for the work probability distributions along the forward and reversed processes, respectively. This relation relates the probability $P_{F}$, of the dissipation function for a trajectory under the influence of an external field in the forward direction, to the probability $P^{R}$, of the dissipation function for a trajectory in the reverse direction. Crooks relation indicates that the probability to dissipate a given amount of work along the forward direction is greater than the probability to absorb the same amount of work along the reverse direction [2-5].

\section{Meaning of work fluctuation}

Figures 1(a) and 1(b) depict, respectively, the schematic and state diagrams of a system exchanging energy and work with the surroundings, first through a process from an initial state A to a final state B, and then through a reverse process moving the system back from state $B$ to its initial state A. In the forward direction from A to B the system receives work from, and rejects heat to, the surroundings; in contrast, in the reverse process from B to A, the system does work on, and receives heat from, the surroundings. There is one equilibrium path but several non-equilibrium trajectories that the system can take when changing state. Therefore, fluctuations within and/or outside the system promote the displacement from an equilibrium path to a non-equilibrium one.

It is important to note that the work produced by the system $\left(W_{s y s \rightarrow}\right)$ is different from the work received by the surroundings $\left(W_{\text {surr } \rightarrow}\right)$ with the difference being equal to the variation in the kinetic energy of the moving boundary. This difference is also equal to the minimum work required to keep the system out of equilibrium. If the difference is equal to zero, then the system is in equilibrium with the surroundings. 


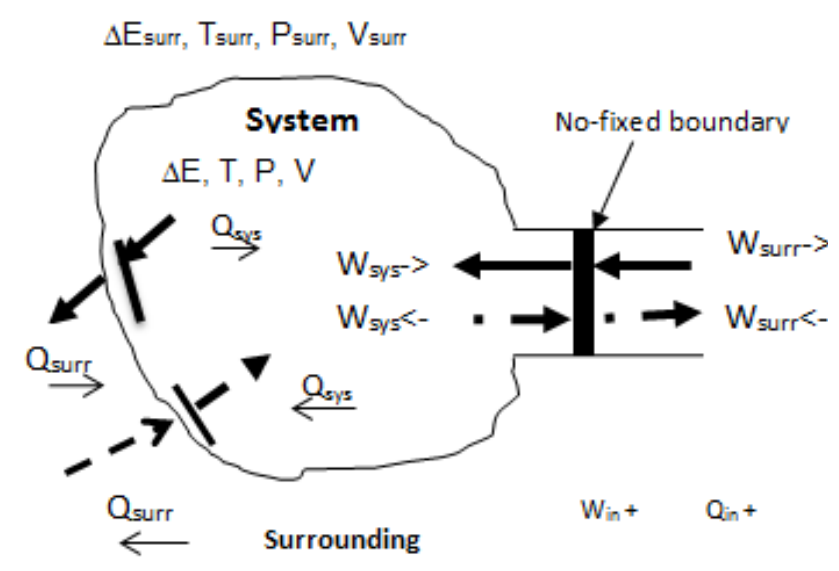

(a)

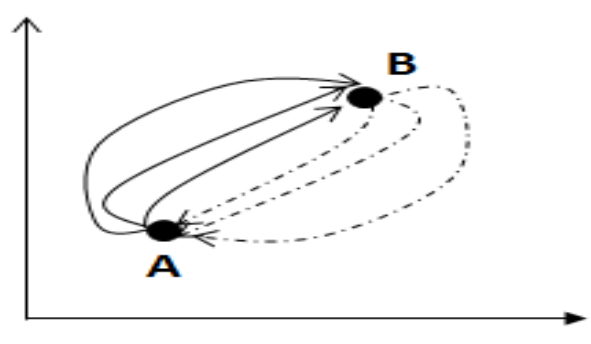

(b)

Figure 1. (a) Schematic diagram and (b) state diagram of a system exchanging work and heat with the surroundings in the forward (from A to B: solid line) and reverse (from $B$ to A: dashed line) directions.

In the forward direction, work should be done on the system to move it from equilibrium state A. As the system starts moving away from equilibrium, a spectrum of fluctuations of different magnitudes are developed; this can mathematically be described by a probability distribution function. In this sense, the variation in the total amount of entropy generation causes variation in the work transferred; consequently, the work needed to keep the system out of equilibrium is also a fluctuating quantity with its amplitude increasing as the system is driven farther away from equilibrium.

Using the first law of thermodynamics, the energy changes inside the system and in the surroundings are respectively written as

$$
\begin{aligned}
& \Delta E_{\text {sys }}=W_{\text {sys } \rightarrow}-Q_{\text {sys } \rightarrow}-P \Delta V_{\text {surr }} \\
& \Delta E_{\text {surr }}=-W_{\text {surr } \rightarrow}+Q_{\text {surr } \rightarrow}+P \Delta V_{\text {surr }}
\end{aligned}
$$

Denoting the fluctuations in work $\left(W_{\rightarrow}^{\prime}\right)$ as the difference between work received by the system minus the work done by the surroundings $\left(W_{\rightarrow}^{\prime}=W_{s y s \rightarrow}-W_{\text {surr } \rightarrow}\right)$, calling fluctuations in heat flow $\left(Q_{\rightarrow}^{\prime}\right)$ the difference between the heat absorbed by the surroundings and the heat released by the system $\left(Q_{\rightarrow}^{\prime}=Q_{\text {sys } \rightarrow}-Q_{\text {surr } \rightarrow}\right)$, and adding Eqns (8) and (9), the total change in energy for the system and its surroundings is found as
$\Delta E_{\text {total }}=W_{\rightarrow}^{\prime}-Q_{\rightarrow}^{\prime}$

It is important to note that whereas $W_{s y s \rightarrow}$ and $Q_{\text {surr } \rightarrow}$ are always positive and $Q_{s y s \rightarrow}$ and $W_{\text {surr } \rightarrow}$ are always negative during the forward path, $W_{\rightarrow}^{\prime}$ and $Q_{\rightarrow}^{\prime}$ can be positive or negative quantities. Applying the second law of thermodynamics on the system and the surroundings, the changes in entropy are obtained as

$\Delta S_{\text {sys }}=-\frac{Q_{\text {sys } \rightarrow}}{T_{\text {surr }}}+S_{g e n}^{\text {sys }} \rightarrow$

$\Delta S_{\text {surr }}=\frac{Q_{\text {surr } \rightarrow}}{T_{\text {surr }}}+S_{\text {gen } \rightarrow}^{\text {surr }}$

By adding Eqns (11) and (12), the total dissipation $\psi_{\rightarrow}$ in the forward process is found to be

$T_{\text {surr }} \Delta S_{\text {total }}=T_{\text {surr }} S_{\text {gen } \rightarrow}^{\text {total }}-Q_{\rightarrow}^{\prime}=\psi_{\rightarrow}$

It can be demonstrated that this work is equal to the deviation of the total entropy from its equilibrium state, $\left(\Delta S_{\text {total }}\right)$, required to keep the system out of equilibrium [18]. This $\left(\Delta S_{\text {total }}\right)$ is different than the $\Delta S_{\text {sys }}$ term, which corresponds to the change in entropy of the system between the equilibrium states $\mathrm{A}$ and $\mathrm{B}$.

If the system and surrounding temperatures are equal $\left(T_{\text {surr }}=T_{\text {sys }}\right)$ then fluctuation in heat is nonexistent (i.e. $Q_{\rightarrow}^{\prime}=0$ ) and if extreme conditions are assumed then fluctuations in work $\left(W_{\rightarrow}^{\prime}\right)$ are completely dissipated (i.e. $\left.W_{\rightarrow}^{\prime}=T_{\text {surr }} S_{\text {gen } \rightarrow}^{\text {total }}\right)$. Setting $Q_{\rightarrow}^{\prime}=0$ in Eqns (10) and (13), then the total entropy change, which includes the entropy of the system $\left(\Delta S_{s y s}\right)$ in addition to the entropy change of the surroundings, is found to be

$\Delta S_{\text {total }}=\frac{\Delta E_{\text {total }}}{T_{\text {surr }}}=\frac{\psi_{\rightarrow}}{T_{\text {surr }}}=\frac{W^{\prime}}{T_{\text {surr }}}$

This indicates that the fluctuation in work is proportional to entropy generation. At steady state we have minimum entropy generation; therefore $\left(W_{\text {sys } \rightarrow}-W_{\text {surr } \rightarrow}\right)=\left(T_{\text {surr }} S_{\text {gen } \rightarrow}^{\text {total }}\right)$. On the other hand, to maintain the system out of equilibrium (see Fig. 2) the work required in general is $\left(W_{\text {sys } \rightarrow}-W_{\text {surr } \rightarrow}\right)=\Delta E_{\text {total }}$ [Eqn (10)]. This means that work is needed to move the system from its equilibrium state and that this work is related to the total entropy generation [Eqn (14)].

By repeating the above process (of changing the system state from A to B several times), different values of $W_{\rightarrow}^{\prime}$ are obtained, because the various experiments are not guaranteed to follow the same path. Therefore, there are several trajectories (Fig. 1b) along which the system is moved from state A to B. Each path is associated with a value for entropy generation and the collection of these 
values can be represented by a distribution function $P$, which according to the Boltzmann postulate is given by

$$
P_{F}\left(\psi_{\rightarrow}\right)=A e^{\frac{\psi_{\rightarrow}}{k_{B} T_{\text {surr }}}}
$$

Therefore, for processes without heat fluctuation, the following applies:

$$
P_{F}\left(W_{\rightarrow}^{\prime}\right)=A e^{\frac{W_{\rightarrow}^{\prime}}{k_{B} T_{\text {surr }}}}
$$

where $A$ is a constant related to probability distribution at equilibrium or reference state. Eqn (16) indicates that the likelihood of fluctuations in the dissipation of work depends on the magnitude of the fluctuations.

In order to understand the variation of total entropy, reference is made to Figure $2 \mathrm{a}$, which shows the change in entropy as a system is moved away from equilibrium due to the work done on it. When work is applied to the system at equilibrium, the total entropy (system plus surroundings) increases as schematically depicted in Figure 2b, where the increase in entropy is modeled as an increase in the height of the ball while departure from equilibrium is represented by how far the ball is from its equilibrium location at $\mathrm{A}$. The increase in the total entropy of the system is understood as an entropy generation because there is not a net heat transfer; therefore, the work applied is considered to be lost.

Therefore, equation (16) can be written as

$$
P\left(W_{\text {sys } \rightarrow}-W_{\text {surr } \rightarrow}\right)=A e^{\frac{W_{\text {sys } \rightarrow}-W_{\text {surr } \rightarrow}}{k_{B} T_{\text {surr }}}}
$$

\section{New derivation of Crooks relation}

In the reverse direction, the system has to undergo a process in which it is taken from the equilibrium state, B, back to its original state, A. For this to be realized, work has to be done by the system. Again, the work needed to keep the system out of equilibrium is a fluctuating quantity with its amplitude increasing as the system is driven farther away from equilibrium.

Similar to the forward direction, the generated maximum work $\left[-\left(W_{\text {sys } \leftarrow}-W_{\text {surr } \leftarrow}\right)\right]$ that is necessary to move the system from the new equilibrium state $B$ to the initial state A following a trajectory with minimum entropy generation, is obtained as

$$
-\left(-W_{\leftarrow}^{\prime}+Q_{\leftarrow}^{\prime}\right)=-\psi_{\leftarrow}
$$

It is obvious that the total entropy generation/energy dissipation in the forward process is not guaranteed to be equal to total entropy generation in the backward process $\left(S_{\text {gen } \rightarrow}^{\text {total }} \neq S_{\text {gen } \leftarrow}^{\text {total }}\right.$ and $\left.Q_{\rightarrow}^{\prime} \neq Q_{\leftarrow}^{\prime}\right)$. This is due to the fact that the irreversibility of the process is not necessarily the same in both directions. The process under consideration moves the system from state A to state B in the forward direction and then back to state $\mathrm{A}$ in the reverse direction. Therefore the irreversibility is a consequence of small fluctuations that are revealed as the process is repeatedly performed. This means that the repeated processes follow different trajectories because the system is out of equilibrium.

By repeating the above process, different values of $W_{\leftarrow}^{\prime}$ and $Q_{\leftarrow}^{\prime}$ are obtained. The collection of these values can be represented by a distribution function, which according to the Boltzmann postulate can be written as

$$
P\left(\psi_{\leftarrow}\right)=A e^{\frac{\psi_{\leftarrow}}{k_{B} T_{\text {sur }}}}
$$

Therefore, for processes without heat fluctuation, the following should hold:

$$
P_{R}\left(-W_{\leftarrow}^{\prime}\right)=A e^{\frac{-W_{\leftarrow}^{\prime}}{k_{B} T_{\text {sur }}}}
$$

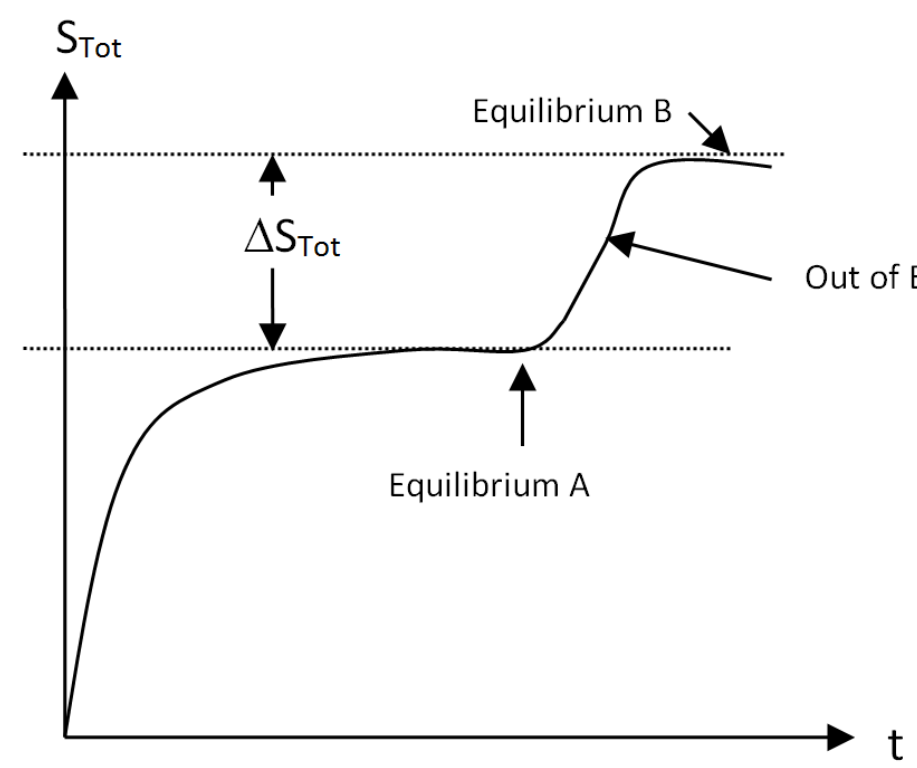

(a)

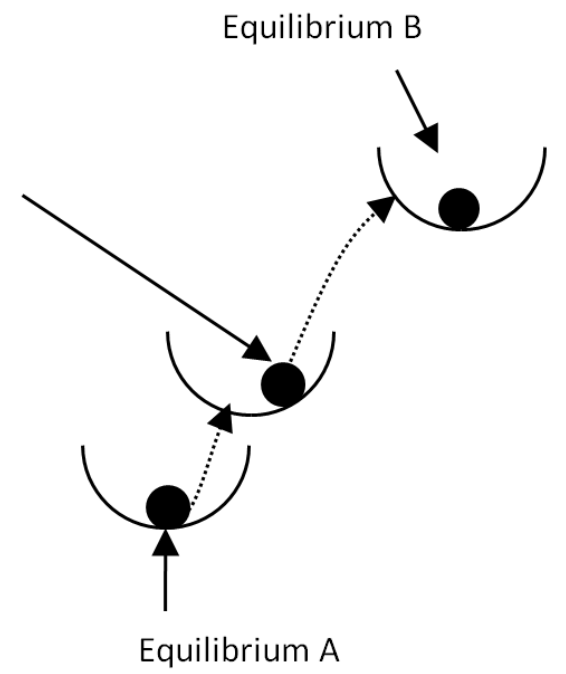

(b)

Figure 2. Schematics of a system showing variation in the total entropy while undergoing a non-equilibrium process. 
The Crooks relation is the ratio of the distribution functions in the forward and backward directions [Eqns (16) and (20)] and can be written as

$$
\frac{P_{F}\left(W_{\rightarrow}^{\prime}\right)}{P_{R}\left(-W_{\leftarrow}^{\prime}\right)}=e^{\frac{W_{\rightarrow}^{\prime}+W_{\leftarrow}^{\prime}}{k_{B} T_{\text {surr }}}}=e^{\frac{S_{\text {gene }}^{\text {total }}}{k_{B}}}
$$

where $S_{g e n}^{\text {total }}=S_{\text {gen } \rightarrow}^{\text {total }}+S_{\text {gen } \leftarrow \text { total }}$. It is easily seen that the reversibility depends on the total energy dissipation. In the absence of any dissipation, the probability for the process to go in either direction is the same. As dissipation of energy increases, irreversibility increases and the probability to go in the forward or reverse directions becomes different. This is the Crooks relation [19], which is a measurement of irreversibility

\section{Closing remarks}

Fluctuations cause a system at equilibrium to simultaneously absorb and reject heat with the net effect being a zero flux. On the other hand, the rates at which a system far from equilibrium absorbs and rejects heat are different. An equation to model these fluctuations was developed by Crooks. In this paper, a simple approach based on a direct application of the first and second laws of thermodynamics along with Boltzmann postulate was followed to derive Crooks relation.

\section{Acknowledgements}

The authors acknowledge the financial support provided by the Universidad Nacional de Colombia and the American university of Beirut

\section{References}

[1] R. Kubo, "The fluctuation-dissipation theorem," Rep. Prog. Phys, vol. 29, pp. 255-284, 1966.

[2] G.E. Crooks, "Nonequilibrium measurements of free energy differences for microscopically reversible Markovian systems" J. Stat. Phys, vol. 90, pp. 14811487, 1998.

[3] G.E. Crooks, "The entropy production fluctuation theorem and the nonequilibrium work relation for free energy differences," Phys. Rev. E., vol. 60 no. 3, pp. 2721-2726, 1999.

[4] G.E. Crooks "Path ensemble averages in systems driven far from equilibrium", Phys. Rev. E., vol. 61, pp. 2361- 2366, 2000.

[5] S.R. de Groot and P. Mazur, Nonequilibrium Thermodynamics, New York: Dover Publication, 1984.

[6] L. Onsager, reprinted in, The collected works of Lars Onsager, Singapore: World Scientific, 1996.

[7] S. Ciliberto, S. Joubaud, and A. Petrosyan, "Fluctuations in out-of-equilibrium systems: from theory to experiment," Journal of Statistical Mechanics: Theory and Experiment, vol. P12003 pp. 1-27, 2010.

[8] R. Van Zon, S. Ciliberto, E. G. D. Cohen, "Power and Heat Fluctuation Theorems for Electric Circuits" Phys. Rev. Lett., vol. 92, pp. 1-7, 2004.
[9] D. J. Evans, E.G.D. Cohen, and G.P. Morris, "Probability of second law violations in shearing steady states," Phys. Rev. Lett, vol. 71, pp. 2401-2404, 1993.

[10] S. Ciliberto and C. Laroche, J. "An experimental test of the Gallavotti-Cohen fluctuation theorem," in International Conference on Disorder and Chaos in honour of Giovanni Paladin Physique. vol. 8 pp. 215219, 1998.

[11]D. J. Evans and D. J. Searles, "Advances in Physics, The fluctuation theorem," vol. 51, pp. 1529-1585, 2002.

[12] D. J. Evans and D. J. Searles, Phys. Rev. "Equilibrium microstates which generate second law violating steady states", vol. 50, pp. 1645-1648, 1994.

[13] G. Gallavotti and E. G.D. Cohen, "Dynamical ensembles in nonequilibrium statistical mechanics" Phys. Rev. Lett., vol. 74, 2694-2697, 1995.

[14] J. Kurchan, "Fluctuation theorem for stochastic dynamics," Journal of Physics A: Mathematical and General, vol. 31, no. 16, pp. 3719-3729, 1998.

[15] Ruelle, D. "Smooth dynamics and new theoretical ideas in nonequilibrium statistical mechanics," Journal of Statistical Physics, vol. 95 (1-2), pp. 393-468, 1999.

[16] Lebowitz, Joel L., and Herbert Spohn. "A GallavottiCohen-type symmetry in the large deviation functional for stochastic dynamics," Journal of Statistical Physics, vol. 95, pp. 333-365, 1999.

[17] Maes, Christian. "The fluctuation theorem as a Gibbs property," Journal of Statistical Physics, vol. 95, pp. 367-392, 1999.

[18] T. Hatano and S. Sasa" Steady-State Thermodynamics of Langevin Systems," Phys. Rev. Lett., vol. 86, pp. 3463-3466, 2001.

[19] R. Van Zon, E. G. D. Cohen, "Stationary and transient work-fluctuation theorems for a dragged Brownian particle," Phys. Rev., vol. 67, pp. 1-20, 2003.

[20]D. Collin, F. Ritort, C. Jarzynski, S. B. Smith, I. Tinoco Jr. and C. Bustamante, "Verification of the Crooks fluctuation theorem and recovery of RNA folding free energies," Nature, vol. 437, pp. 231-234, 2005.

[21]C. Bustamante, J. Liphardt and F. Ritort, "The nonequilibrium thermodynamics of small systems," Phys Today, vol. 58, no. 7, pp. 43-48, 2005.

[22]C. Jarzynski, "Nonequilibrium Equality for Free Energy Differences," Phys. Rev. Lett., vol. 78, pp. 2690-2693, 1997.

[23] C. Jarzynski, "Equilibrium free-energy differences from nonequilibrium measurements: A master-equation approach," Phys. Rev. E., vol. 56, 5018 - 5035, 1997.

[24]D. Chandler, Introduction to Modern Statistical Mechanics, New York: Oxford University Press, 1987. 